important are the proportion of infections that are symptomatic in men and women; duration of untreated infection; and incidence of PID, infertility and ectopic pregnancy attributable to M. genitalium.

Conclusion Further empirical work is required to improve understanding of the key aspects of M. genitalium's natural history which we have identified before it will be possible to determine if screening is cost-effective.

Disclosure of interest statement This presentation reports independent research commissioned by the National Institute for Health Research (NIHR) under its Programme Grants for Applied Research scheme (RP-PG-0707-10208). In addition, PJW thanks the UK Medical Research Council for Centre funding (grant MR/K010174/1) and also thanks the UK National Institute for Health Research Health Protection Research Unit (NIHR HPRU) in Modelling Methodology at Imperial College London in partnership with Public Health England (PHE) for funding (grant HPRU-2012-10080). The views expressed in this paper are those of the authors and not necessarily those of the NHS, the NIHR, the Department of Health, or Public Health England. The funders had no role in study design, data collection and analysis, decision to publish, or preparation of the manuscript. We have no conflicts of interest.

\section{P09.12 APPARENTLY-DIFFERENT CLEARANCE RATES FROM COHORT STUDIES OF MYCOPLASMA GENITALIUM ARE CONSISTENT AFTER ACCOUNTING FOR INCIDENCE OF (RE) INFECTION AND STUDY DESIGN}

${ }^{1,2} \mathrm{~T}$ Smieszek, ${ }^{1,2} \mathrm{PJ}$ White* ${ }^{1}$ Modelling and Economics Unit, Centre for Infectious Disease Surveillance and Control, Public Health England, London, UK; ${ }^{2}$ NIHR Health Protection Research Unit in Modelling Methodology and MRC Centre for Outbreak Analysis and Modelling, Department of Infectious Disease Epidemiology, School of Public Health, Imperial College London, UK

\subsection{6/sextrans-2015-052270.396}

Introduction Mycoplasma genitalium is increasingly recognised as an important cause of urethritis, cervicitis, PID, infertility, and increased HIV risk. A better understanding of its natural history is crucial to informing control policy. Two cohort studies (students in London, UK, and sex workers in Uganda) suggest very different clearance rates. We aimed to gain insight into the reasons and to obtain improved estimates by making maximal use of the data from those studies.

Methods To estimate incidence and clearance rates, we developed a model for time-to-event analysis incorporating the processes of individuals becoming infected after enrolment into the study, clearing infection, becoming reinfected after clearance, and fitted it to data from the two cohort studies. As the studies collected limited data on sexual partnership dynamics, we tested the sensitivity of the model to different assumptions that were consistent with the available information. Additionally, we modelled study-design differences, including sample handling conditions affecting testing sensitivity.

Results In the London students, the estimated clearance rate was 0.80p.a. (mean duration 15 months), with incidence $1.31 \%$ p.a. and $3.93 \%$ p.a. (in low- and high-risk groups, respectively). Without adjusting for study design, corresponding estimates from the Ugandan data were 3.35p.a. (mean duration 3.6 months) and $44 \%$ p.a. Clearance-rate differences could be explained by differences in testing sensitivity, with 'true' rates being similar, and adjusted incidence in the Uganda study being $21 \%$ p.a.
Conclusion Analysis needs to account for study design, and we recommend cohort studies collect more information on partnership dynamics to inform more-accurate estimates of natural-history parameters. The cohorts' clearance rates were probably similar, with the apparent difference due mostly to differences in sample handling in the studies, and perhaps partly due to the sex workers having more-frequent antibiotic treatment (for other infections), and in the London students some reinfection in stable partnerships causing some of the apparently-persistent infection.

Disclosure of interest statement TS and PJW thank the UK National Institute for Health Research Health Protection Research Unit (NIHR HPRU) in Modelling Methodology at Imperial College London in partnership with Public Health England (PHE) for funding (grant HPRU-2012-10080). PJW also thanks the UK Medical Research Council for Centre funding (grant MR/K010174/1). The views expressed are those of the authors and not necessarily those of the NHS, the NIHR, the Department of Health, or Public Health England. We have no conflicts of interest.

\section{P09.13 IMPACT AND COST-EFFECTIVENESS OF POINT-OF-CARE TESTING FOR CHLAMYDIA: ACCOUNTING FOR GEOGRAPHIC VARIATION IN INFECTION BURDEN AND TESTING RATES, HEALTH SERVICE CONFIGURATION, AND IMPLEMENTATION STRATEGY}

${ }^{1,2,3,4} \mathrm{CE}$ Dangerfield, ${ }^{1,2,3} \mathrm{E}$ Sherrard-Smith, ${ }^{2,3} \mathrm{~N}$ Green, ${ }^{1} \mathrm{E}$ Harding-Esch, ${ }^{1} \mathrm{R}$ Howell-Jones, ${ }^{5}$ Y Choi, ${ }^{1,6} \mathrm{CM}$ Lowndes, ${ }^{2,3} \mathrm{PJ}$ White*. ${ }^{1}$ HIV \& STI Department, Centre for Infectious Disease Surveillance and Control, Public Health England, London, UK; ${ }^{2}$ Modelling \& Economics Unit, Centre for Infectious Disease Surveillance and Control, Public Health England, London, UK; ${ }^{3}$ MRC Centre for Outbreak Analysis and Modelling and Health Protection Research Unit in Modelling Methodology, Department of Infectious Disease Epidemiology, Imperial College London, UK; ${ }^{4}$ Department of Plant Sciences, University of Cambridge, UK; ${ }^{5}$ Immunisation Department, Centre for Infectious Disease Surveillance and Control, Public Health England, London, UK; ${ }^{6}$ London School of Hygiene and Tropical Medicine, UK

\subsection{6/sextrans-2015-052270.397}

Introduction Point-of-care tests (PoCTs) for Chlamydia trachomatis potentially improve control by reducing transmission through reduced treatment delay, reduced loss to follow up, and greater convenience for patients leading to increased testing. However, with $\sim 3-5$ fold variation in rates of Chlamydia testing and diagnosis across geographic settings in England, in assessing cost-effectiveness the epidemiological context needs to be considered.

Methods We developed a transmission-dynamic model to capture geographic variation in rates of testing and diagnosis using current technology to allow assessment of the impact of implementing PoCTs in different clinical services in different localities. The model incorporates heterogeneity in sexual partner change rates and is stratified by age and sex. It uses behavioural and prevalence data from the Natsal national survey, and Public Health England surveillance data on testing and diagnosis rates. Uncertainty in natural history and behavioural parameters is captured by Monte Carlo methods. Health service reconfiguration using PoCT is considered, including rates of PoCT introduction and reduction in presumptive treatment.

Results The model captures observed geographic variation in rates of testing and diagnosis in females and males, which affects the impact and cost-effectiveness of PoCT introduction. In general, whilst PoCTs may reduce incidence by increasing diagnosis 
and treatment, if sensitivity is lower than conventional testing then there is potentially increased transmission from false-negative patients. The net effect depends upon characteristics of the particular PoCT.

Conclusion PoCT sensitivity and specificity are key determinants of whether replacing conventional testing is likely to reduce or increase the incidence of infection. Importantly, if the convenience of PoCT increases testing rates then diagnoses might initially increase even though incidence falls; interpretation of surveillance data needs to account for this. This work is the foundation of a user-friendly web-based tool assessing the (cost-) effectiveness of introducing PoCTs in different local settings. Disclosure of interest statement CED, ESS, NG, EHE, RHJ, YC, CML, and PJW thank Innovate UK for funding. PJW thanks the UK Medical Research Council for Centre funding (grant MR/ K010174/1), and also thanks the UK National Institute for Health Research Health Protection Research Unit (NIHR HPRU) in Modelling Methodology at Imperial College London in partnership with Public Health England (PHE) for funding (grant HPRU-2012-10080). The funding sources had no involvement in the study design or conduct; the collection, analysis and interpretation of data; the preparation, review or approval of the manuscript; or the decision to submit the manuscript for publication. The views expressed are those of the authors and not necessarily those of the NHS, the NIHR, the Department of Health, or Public Health England. CED was employed for part of the project by the National Chlamydia Screening Programme. The other authors declare no competing interests.

\section{P09.14 IMPLEMENTATION OF ORAL AND SELF-COLLECTED RECTAL SWABS FOR N. GONORRHOEAE AND C. TRACHOMATIS DETECTION AS A COMPONENT OF LOCAL HEALTH DEPARTMENT OUTREACH TESTING}

${ }^{1} \mathrm{BC}$ Barr, ${ }^{2} \mathrm{~N}$ Nall, ${ }^{1,2} \mathrm{CJ}$ McNeil, 'LH Bachmann ${ }^{*} .{ }^{1}$ Wake Forest University Health Sciences, Winston-Salem, NC, USA; ${ }^{2}$ Forsyth County Department of Public Health, Winston-Salem, NC, USA

\subsection{6/sextrans-2015-052270.398}

Introduction The availability of extra-genital nucleic acid amplification testing (NAAT) for gonorrhoea (GC) and chlamydia (CT) is limited in many clinical and outreach settings. A recent initiative in Forsyth County, NC funded NAAT testing at oropharyngeal and rectal sites in both of these settings.

Methods A retrospective chart review was conducted for all males and females aged 12-80 who reported to an outreach site or STD clinic (MSM only) January 1, 2014 to February 28, 2015.

Results Clinic: 131 rectal, 163 pharyngeal, and 176 urethral NAAT tests were performed on 181 males. Twenty-five rectal (19.1\%), 22 pharyngeal (13.5\%), and $22(12.5 \%)$ urethral specimens were GC positive. 24 rectal $(18.3 \%)$ and 7 urethral $(4.0 \%)$ were CT positive. 5 rectal GC $(20.0 \%), 8$ pharyngeal GC (36.4\%), and 13 rectal CT (54.2\%) infections would have been missed in the absence of extra-genital testing.

Outreach 47 rectal, 157 pharyngeal, and 162 urogenital NAATs were collected. For GC, 3 rectal (6.4\%), 3 pharyngeal (1.9\%), and 2 urogenital (1.2\%) specimens were positive. Six (12.8\%) and $9(5.6 \%)$ individuals were positive for rectal and urogenital CT, respectively. 2 rectal GC (66.7\%), 3 pharyngeal GC (100\%), and 4 rectal CT (66.7\%) infections would have been missed without extra-genital NAAT.
When NAAT and bacterial culture for GC were run on the same samples from clinic patients, culture detected $48 \%(12 / 25)$ of the rectal and $45.2 \%(10 / 22)$ of the pharyngeal infections detected by NAAT.

Conclusion A significant prevalence of extra-genital GC and CT infections were noted in both the clinic and outreach populations. In the absence of NAAT-based extra-genital testing approximately half of rectal and pharyngeal GC infections would have been missed with culture and more than half of the rectal CT infections would have been missed. Assurance of access of NAAT-based extra-genital testing is critical for STI control efforts.

\section{P09.15 TIME TO CLEARANCE FOR MOLECULAR TEST-OF-CURE AMONG MEN TREATED FOR URETHRAL, PHARYNGEAL, OR RECTAL GONORRHOEA IN SAN FRANCISCO, 2013-2014}

TQ Nguyen*, SE Cohen, T Noohi, RP Kohn, SS Philip. San Francisco Department of Public Health

\subsection{6/sextrans-2015-052270.399}

Introduction Due to the potential emergence of cephalosporinresistant Neisseria gonorrhoeae (NG), some public health agencies recommend a test of cure (TOC) using nucleic acid amplification tests (NAATs). NAATs can remain positive after the infection has been effectively eradicated; the optimal time to obtain a TOC is unclear. To inform TOC recommendations, we conducted NAATs for 21 days following treatment for men who have sex with men (MSM) NG-infected at the urethra, rectum or pharynx.

Methods MSM with untreated urethral, rectal or pharyngeal gonorrhoea were eligible. At enrollment, prior to provision of antibiotics, infection-site specimens were collected by a clinician for culture and NAAT testing. Participants whose enrollment-day specimens were negative were excluded. Participants self-collected daily infection-site specimens (rectal or pharyngeal swabs or urine) for 3 weeks and returned on days 7, 14, and 21 to submit their self-collected specimens for NAAT testing and provide clinician-collected specimens for culture and NAAT testing. We planned to follow at least 10 participants for at least 7 days in each of the urethral $(\mathrm{U})$, rectal $(\mathrm{R})$, and pharyngeal $(\mathrm{P})$ study arms.

Results We enrolled 46 MSM. Eleven participants had negative enrollment-day specimens, and 4 did not return after enrollment. Of the 31 included in the analysis (11U, 11R, 9P), $22(71 \%)$ provided all 21 days of specimens; another 6 provided nearly all study specimens. The majority $(94 \%)$ were negative on Day 7 $(11 \mathrm{U}, 11 \mathrm{R}, 7 \mathrm{P})$, though $4(2 \mathrm{U}, 1 \mathrm{R}, 1 \mathrm{P})$ had subsequent positive results.

Conclusion A high proportion of participants had a negative NAAT before antibiotics, despite a prior positive result, indicating either an original false positive result or natural clearance of infection. Additional analyses will determine the effectiveness of other TOC time-points, including median time to: first negative result, 3 consecutive negative results, and clearance (consistently negative results through follow-up); and clearance at Days 14 and 21.

Disclosure of interest statement No pharmaceutical grants were received in the development of this study. 